\title{
Risk Assessment and Economic Impact Analysis of the Implementation of New European Legislation on Radiopharmaceuticals in Italy: The Case of the New Monograph Chapter Compounding of Radiopharmaceuticals (PHARMEUROPA, Vol. 23, No. 4, October 2011)
}

\author{
Chittò G, Di Domenico E, Gandolfo P, Ria F*, Tafuri C, and Papa S.
}

Diagnostic Imaging Department, CDI Centro Diagnostico Italiano S.p.A., 20 via Saint Bon, 20147, Milano, Italy, and Quality Department, CDI Centro Diagnostico Italiano S.p.A., 20 via Saint Bon, 20147, Milano, Italy

\begin{abstract}
An assessment of the new monograph chapter Compounding of Radiopharmaceuticals has been conducted on the basis of the first period of implementation of Italian legislation on Good Radiopharmaceuticals Practice (NBP) in the preparation of radiopharmaceuticals, in keeping with Decree by the Italian Ministry of Health dated March 30, 2005. This approach is well grounded in the several points of similarity between the two sets of regulations. The impact on patient risk, on staff risk, and on healthcare organization risk, has been assessed. At the same time, the actual costs of coming into compliance with regulations have been estimated.

A change risk analysis has been performed through the identification of healthcare-associated risks, the analysis and measurement of the likelihood of occurrence and of the potential impact in terms of patient harm and staff harm, and the determination of the healthcare organization's controlling capability. In order to evaluate the economic impact, the expenses directly related to the implementation of the activities as per ministerial decree have been estimated after calculating the overall costs unrelated to NBP implementation. The resulting costs have then been averaged over the total number of patient services delivered.

NBP implementation shows an extremely positive impact on risk management for both patients receiving Nuclear Medicine services and the healthcare organization. With regard to healthcare workers, instead, the implementation of these regulations has a negative effect on the risk for greater exposure and a positive effect on the defense against litigation. The economic impact analysis of NBP implementation shows a $34 \%$ increase in the costs for a single patient service.

The implementation of the ministerial decree allows for greater detectability of and control over a number of critical elements, paving the way for risk management and minimization. We, therefore, believe that the proposed tool can provide basic criteria for analysis that could be used by other organizations setting about completing the same process.
\end{abstract}

Keywords: Quality risk management, Quality control, Compounding of radiopharmaceuticals, Risk assessment, Economic Evaluation.

\section{INTRODUCTION}

The monograph chapter Compounding of radiopharmaceuticals (PHARMEUROPA, Vol. 23, No.4, October 2011) was preceded, in Italian legislation, by the Ministry of Health's Decree dated March 30, 2005 - Good Radiopharmaceuticals Practice (NBP) in the preparation of radiopharmaceuticals in Nuclear Medicine (NM). These two sets of regulations have many points in common, and the purpose of this study is to represent all the necessary costs required for ensuring greater quality of radiopharmaceuticals as well as the positive impact on both patient risk and organizational risk assessment in the first period of NBP implementation in the CDI Nuclear Medicine unit. The data reported may help other healthcare organizations that must embark on a similar course of action in order to come into compliance with both Italian and European regulations.

*Address correspondence to this author at the CDI Centro Diagnostico Italiano S.p.A., 20 via Saint Bon, 20147 Milano, Italy; Tel: +39 02 48317025; Fax: -----------; E-mail: francesco.ria@cdi.it
Introducing a quality system in a Nuclear Medicine unit permits the use of new quality tools. For example, the risk assessment allows to consider all critical factors related to the processes. From this assessment arise all actions necessary to mitigate the index risk, new goals for quality improvement and new process indicators.

The implementation of a quality system requires an add cost in terms of human resources (new responsibilities assigned to the staff) and an economic one (costs related to raw materials and equipment).

In particular, NBP implementation offers an opportunity to manage the risks arising from the use of radiopharmaceuticals, thus improving overall impact and ensuring increased safety for patients, staff, and healthcare organizations. In particular, positive effects are attained in terms of greater detectability of the potential harm to patient health resulting from the administration of unnecessary doses (radionuclidic and radiochemical purity) and of radiopharmaceuticals that could fail to meet the required sterility and apyrogenicity criteria. Greater detectability of and control over the above- 
mentioned critical elements lead to minimizing any resulting risk and/or potential harm, as described by the change risk analysis.

The actual costs incurred for coming into compliance with regulations will be highlighted versus the overall management costs of a nuclear medicine unit, thus offering a tool that may facilitate a more accurate analysis of economic impact. Moreover, the study will show the incidence on the organizational cost of a single patient service following the introduction of a quality management system.

\section{MATERIALS AND METHODS}

\subsection{Risk Assessment}

Conducting risk analysis within a healthcare organization does not only involve considering the potential to harm the patient, that is undoubtedly the most important risk, but it also requires an evaluation of every possible negative impact on both healthcare workers and on the hospital organization as a whole.

All risks associated with each and every activity or process need to be analyzed, assessed, communicated, eliminated and monitored. The starting point is represented by risk assessment that consists in several steps:

- identification of the intrinsic risks of a healthcare activity;

- analysis and measurement of the likelihood of occurrence and of the potential impact in terms of patient/staff harm;

- determination of the grade of organizational controlling ability;

- opportunity assessment in terms of risk/benefit and cost/benefit ratios.

The risk management process is subdivided into four key operational phases:

1. risk identification, aimed at identifying all risks faced by an organization;

2. risk assessment and analysis, the purpose of which is to determine the likelihood and expected severity (magnitude) associated with the occurrence of harmful events;

3. risk planning, that identifies the most appropriate actions to be taken in order to reduce risk;

4. process monitoring.

The implementation of Good Radiopharmaceuticals Practice (NBP) in the preparation of radiopharmaceuticals in $\mathrm{Nu}$ clear Medicine, thus allows an organization to have very good solutions already in place for minimizing the risks identified in the first two steps of risk management.

The relative risk of threat, to be understood as anticipated harm resulting from an uncertain event, is the product of the severity of injuries (harm) by the relative likelihood of occurrence:

$$
\text { Risk }=\text { Likelihood }(\mathrm{P}) \times \text { Severity }(\mathrm{G})
$$

Likelihood (P) is given by an estimate of the possibility of a critical event occurring, whereas severity is given by:
Severity = magnitude of potential harm $(\mathrm{D})-$ relative mitigation (A)

This means that the severity of any potential harm is not only an index of how negatively an event has affected or could affect a hospital organization, but also an index of how largely it can be mitigated by all internal and/or external defense mechanisms that are implemented.

Consequently, the risk index for a given event is inversely proportional to an organization's capability to mitigate harm.

In detail, in order to be able to assign a value to the magnitude or size of potential harm, it is necessary to consider the impact on individuals (IP), the impact on property (IB), and the impact on work/performance (IL). Therefore, the size of harm (D) will be given by the sum of the values assigned to these three parameters.

Relative mitigation (A), that stands for an organization's capability for internal and/or external defense, is also given by the sum of three parameters: preparedness (RP), internal response (RI), and external response (RE). In other words, mitigation considers the planning for actions to prevent a threat from occurring, the effective response time and resources that an organization can deploy when a threat occurs, and, finally, the aids and supplies that can come from outside an hospital organization to try and curb an event after it has occurred.

In short, the relative threat $(\mathrm{R})$, which is an index of risk, is obtained from the following formula:

$$
R=\frac{P}{X}\left(\frac{I P+I B+I L+R P+R I+R E}{6 X}\right)
$$

where $\mathrm{X}$ is the maximum scale value that can be assigned to the above parameters.

Mitigation parameters are also added up in the formula, because a reversed value scale versus the scale used for magnitude parameters has been chosen in order to evaluate their positive impact on relative threat.

The study reviews the relative threats to staff, patients, and organizations, thereby considering all aspects that can harm all of them. The choice was made to use an assessment scale comprised of three values plus one that indicates non applicability of a criterion.

Scale for magnitude parameters (IP, IB, IL)

$0=$ Non Applicable

$1=$ low $\backslash$ none (0-2 times a year)

$2=$ moderate (2-5 times a year)

3 = highlabsolute (> 5 times a year)

Scale for mitigation parameters (RP, RP, RE)

$0=$ Non Applicable

1 = highlabsolute

$2=$ moderate

$3=$ low $\backslash$ moderate 
Table 1. Summary of Fixed Costs of a Nuclear Medicine Unit and of the Costs Relating Only to the Implementation of the New Legislation

\begin{tabular}{|c|c|}
\hline Nuclear Medicine Fixed Costs & $\begin{array}{c}\text { Costs Relating to the Implementation of NBP in the Preparation of } \\
\text { Radiopharmaceuticals }\end{array}$ \\
\hline Medical staff ( 1 employed, 1 contracted) & Purchase of QC reagents \\
\hline $\begin{array}{l}\text { Tomography equipment (Annual depreciation expense. No cost } \\
\text { after depreciation period has expired) }\end{array}$ & $\begin{array}{l}\text { Annual cost of medical staff hours dedicated to activities concerning NBP-NM } \\
\text { (15\% of total hours) }\end{array}$ \\
\hline Tomography equipment maintenance & $\begin{array}{l}\text { Annual cost of Radiology Technicians' hours dedicated to activities concerning } \\
\text { NBP-NM ( } 20 \% \text { of total hours) }\end{array}$ \\
\hline Radiology Technicians ( 2 employed, 1 contracted) & Microbiological monitoring and particle monitoring (environment and staff) \\
\hline Nursing Staff (1 contracted) & Equipment qualifications \\
\hline \multirow[t]{6}{*}{ Purchase of drugs and Tc $99 \mathrm{~m}$ generator } & Maintenance contracts \\
\hline & $\begin{array}{l}\text { Equipment (annual depreciation expense for chromatography apparatus, contain- } \\
\text { ment cells, dose calibrators, pass through box, solvent cabinet) }\end{array}$ \\
\hline & Design \\
\hline & $\begin{array}{l}\text { Construction and renovation works (annual amortization cost for construction and } \\
\text { renovation works, electrical system, ventilation, air extraction and air conditioning } \\
\text { [HVAC] system) }\end{array}$ \\
\hline & Consultation for the implementation of a quality management system \\
\hline & Any additional expenses for re-compounding due to nonconforming preparation \\
\hline
\end{tabular}

This choice is mandated by the diversity of threats that may affect patients, staff, and organizations, and that are highly heterogeneous by nature, suggesting to avoid using overly complex assessment scales.

It goes without saying that the method proposed here can be applied to more homogeneous situations allowing parameters to be assessed in greater detail.

\subsection{Cost Assessment}

In order to assess the economic impact of the implementation of NBP in the preparation of radiopharmaceuticals in $\mathrm{NM}$, the fixed costs $\left(\mathrm{C}_{\mathrm{f}}\right)$ of a nuclear medicine unit have been estimated, i.e. all costs unrelated to the new legislation. Then the expenses exclusively relating to the activities introduced following the enforcement of Ministerial Decree of 30 March 2005, have been estimated $\left(\mathrm{C}_{\mathrm{NBP}}\right)$. Considering $\mathrm{N}$ as the total number of patient services delivered in one year, the average cost of a single patient service is given by

$$
C_{M}=\frac{C_{f}+C_{N B P}}{N}
$$

The average cost of implementing NBP in the preparation of radiopharmaceuticals in NM is given by

$$
C_{L}=\frac{C_{N B P}}{N}
$$

It follows that the percentage increase in the expenses incurred in a nuclear medicine unit is given by

$$
C_{\%}=\frac{C_{L}}{C_{M}} \times 100
$$

The analysis has considered exclusively the activities relating to scintigraphy studies using Technetium-99m (Tc99m). Depreciation years have been considered to be seven.

\section{RESULTS AND DISCUSSION}

Risk assessment is, by its very nature, a subjective activity. The tool proposed here is intended to provide criteria for analysis that can be used in any Nuclear Medicine radiopharmacy (hot lab) with a view to also ensuring the possibility of making comparisons across facilities. In order for risk assessment to be more effective, it has to be conducted by a team involving all professional disciplines working in $\mathrm{Nu}-$ clear Medicine and dealing with quality management in healthcare organizations.

\subsection{Patient Risk}

It has been estimated that the implementation of NBPNM can significantly abate the relative risk to patients. Likely critical events reviewed, including their consequences on patients, are described in the following table:

As it could be expected considering the threats, the implementation of the regulations has a highly positive impact on risk management in patients undergoing nuclear medicine studies.

\subsection{Staff Risk}

With regard to staff working in nuclear medicine units, variation in the level of risk related to NBP-NM implementa- 
Table 2. Patient Risk: Critical Events and their Effects

\begin{tabular}{|l|l|}
\hline \multicolumn{1}{|c|}{ Critical Event } & Effect \\
\hline \hline \multirow{2}{*}{ Thresholds of radiochemical purity of the preparation are exceeded } & $\begin{array}{l}\text { The administered dose of radiopharmaceutical is not entirely useful for } \\
\text { diagnostic purposes. }\end{array}$ \\
\cline { 2 - 3 } & Lesser quality of the study \\
\hline Preparation sterility and apyrogenicity are not assured & Patient infection \\
\hline $\begin{array}{l}\text { Staff are not trained / are inadequately trained according to established and } \\
\text { shared procedures }\end{array}$ & High variability in staff behavior \\
\hline The radiopharmaceutical preparation process is not tracked & Lower safety of both process and diagnostic result \\
\hline The radiopharmaceutical administration process is not tracked & \\
\hline
\end{tabular}

Table 3. Staff Risk: Critical Events and their Effects

\begin{tabular}{|l|l|}
\hline \multicolumn{1}{|c|}{ Critical Event } & \multicolumn{1}{c|}{ Effect } \\
\hline \hline Error during preparation of radiopharmaceutical & Greater exposure to ionizing radiation \\
\hline Litigation & Increase in the costs of defensive medicine \\
\hline
\end{tabular}

Table 4. Organizational risk: critical events and their effects.

\begin{tabular}{|l|l|}
\hline \multicolumn{1}{|c|}{ Critical Event } & Effects \\
\hline \hline Litigation & Increase in the costs of defensive medicine \\
\hline Delivery of below-standard services & Repeat diagnostic studies \\
\hline Staff not trained / inadequately trained & High variability in staff behavior \\
\hline Re-compounding of radiopharmaceutical & More money spent on raw materials \\
\hline Lack of clearly assigned responsibilities & Reduction in the quality of the nuclear medicine diagnostic process \\
\hline
\end{tabular}

tion has been estimated on the basis of the critical events listed in Table 3.

Before the new legislation came into effect, recompounding of radiopharmaceuticals was exclusively due to accidental situations (e.g. breaks). However, performing quality controls on a regular basis allows compounding errors to be identified so that a radiopharmaceutical with analytical values exceeding quality specifications should be reprepared anew prior to administration. This, in turn, exposes staff to greater risk for radiation exposure. On the other hand, however, the new regulations have a positive impact on the risk for litigation involving nuclear medicine staff.

\subsection{Organizational Risk}

Overall, NBP-NM implementation has had a positive impact on organizational risk management, as evaluated on the basis of the following critical events and their consequences on the healthcare organization:

The analysis carried out shows how the risks reported in Table 4 decrease noticeably. The only exception is given by the risk for financial loss resulting from an excessive increase in the costs of raw materials because radiopharmaceuticals have to be prepared again.
Moreover, the organization can benefit from the possibility of obtaining analytical data about the compounding process that allow the whole diagnostic process to be better monitored and assessed. These data can be used to apply the principles of continuous quality improvement summarized in the PDCA (Plan - Do - Check - Act) model.

\subsection{Costs}

The Italian context is not characterized by the presence of centralized radiopharmacy facilities where radiopharmaceuticals are compounded for several hospitals. On the contrary, every nuclear medicine unit has an internal radiopharmacy (hot lab) where Tc99m generator elution is used for radiolabeling compounds. In the light of the new legislation, this implies a considerable increase in costs in comparison with centralized radiopharmacy management.

The costs shown in the chart below have been calculated for a facility having no quality management system in place and performing no quality controls on radiopharmaceuticals at the time when the new regulations are implemented.

Nuclear medicine units that have already started to define their quality control systems, should only consider those cost items for the actions still to be implemented. 


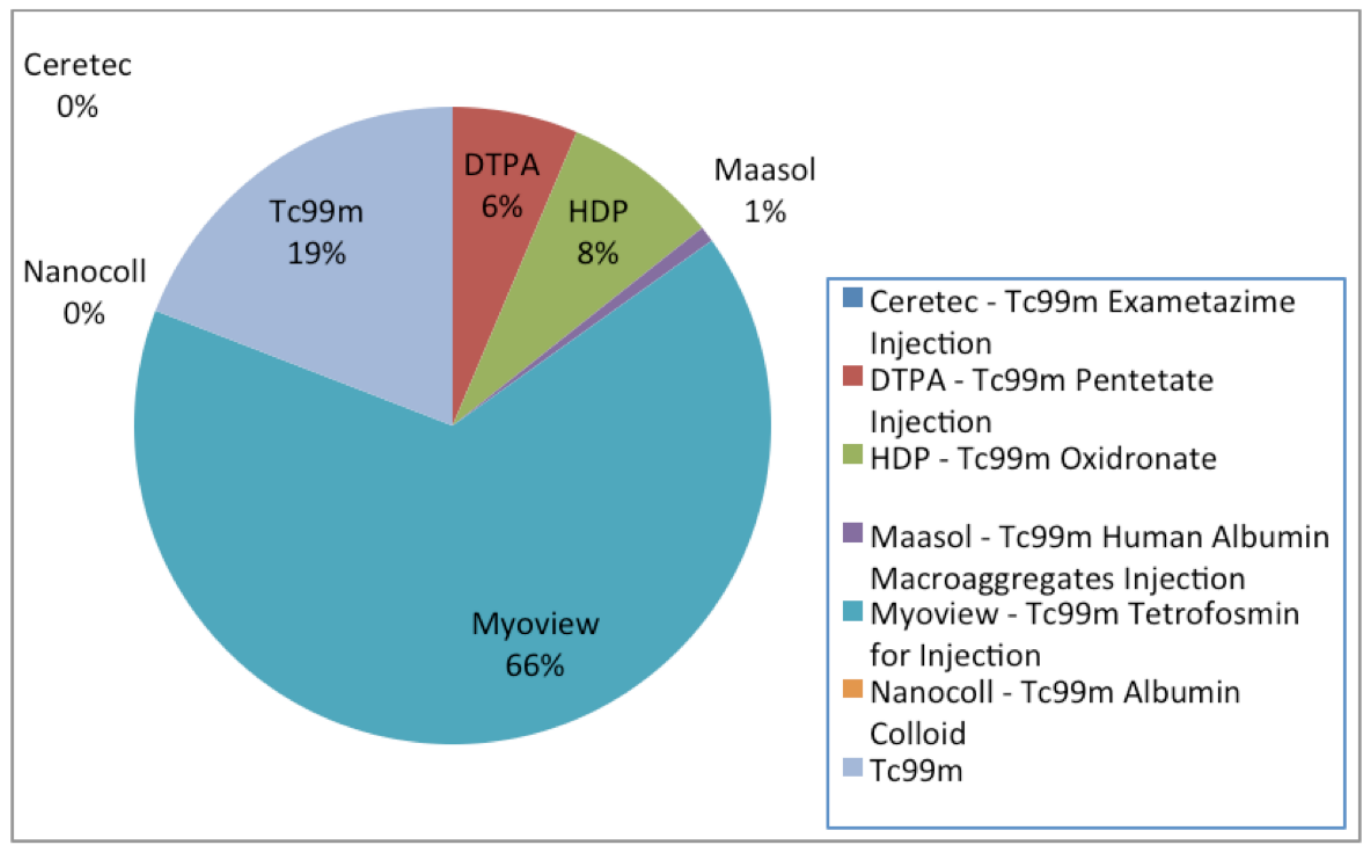

Chart 1. Percentage distribution of drugs purchased in 2012.

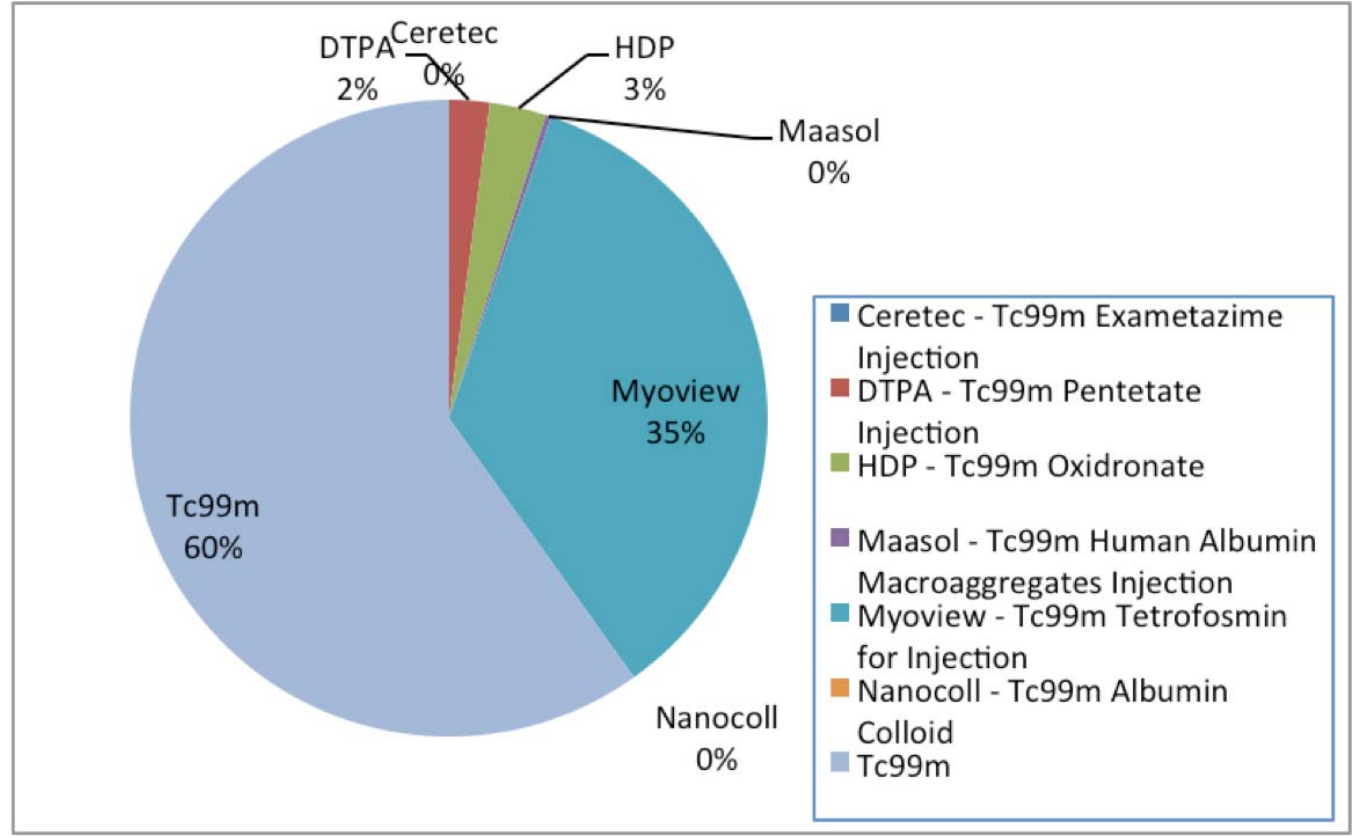

Chart 2. Distribution of the cost for purchasing drugs in 2012.

In the CDI nuclear medicine unit, the percentage distribution and cost of the drugs purchased in 2012 are shown in chart 1 and chart 2, respectively. As it can be noted, $60 \%$ of these costs (accounting for 15\% approximately of overall expenses) is due to the purchase of Tc99m generators which represent a fixed cost for all Nuclear Medicine facilities.

The percentage distribution of the costs for the implementation of the new regulations and the fixed costs of the Nuclear Medicine unit are shown in chart 3 and chart 4, respectively.

In the light of these assessments, it can be concluded that NBP-NM implementation has entailed an approximately $34 \%$ increase in the costs for a single patient service. This calculation applies to a nuclear medicine unit where radio- pharmaceuticals are compounded on its premises, and that would not perform any analytical controls on radiopharmaceuticals and would not work according to any quality management system prior to the enforcement of the new regulations.

\section{CONCLUSION}

Risk assessment conducted by CDI, before and after the implementation of Good Radiopharmaceutical Practice in the preparation of radiopharmaceuticals in Nuclear Medicine, shows that these regulations have a positive impact on the risk indexes for most of the events that could occur in a Nuclear Medicine unit. 


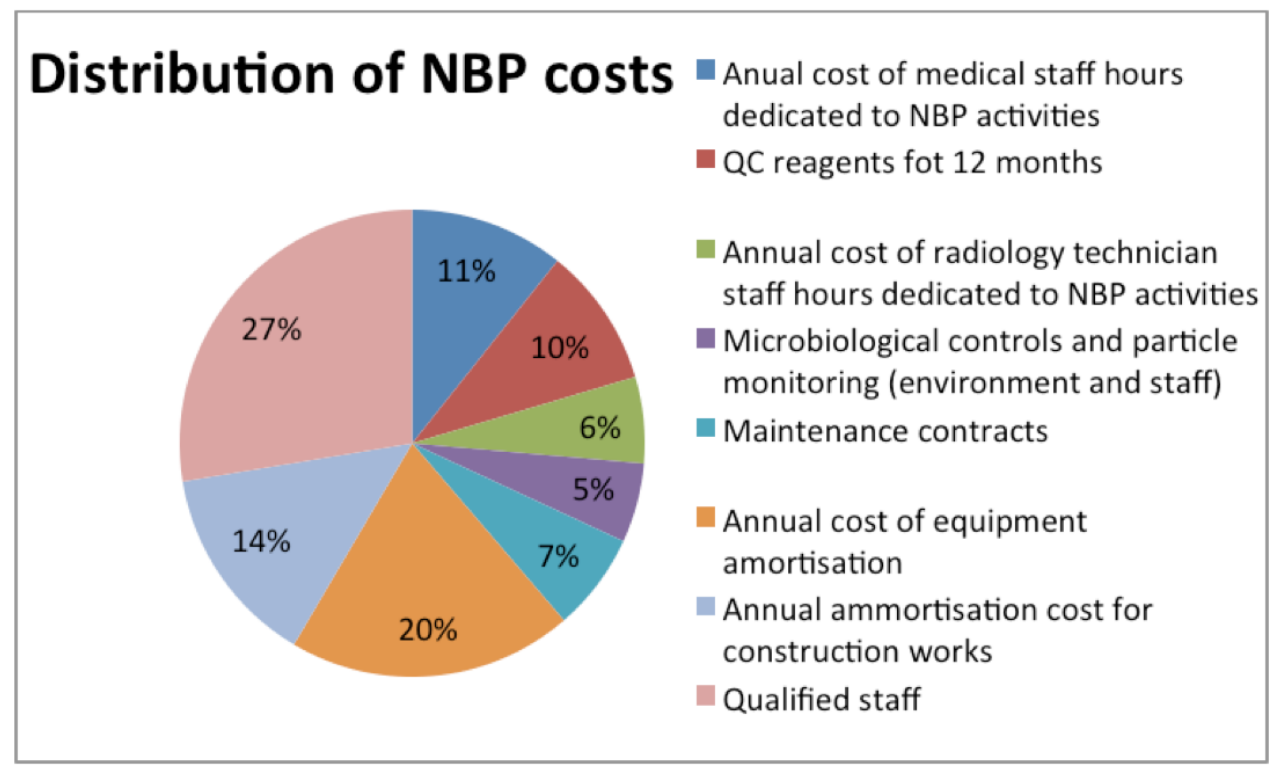

Chart 3. Percentage distribution of the costs for NBP-NM implementation.

\section{Distribution of fixed costs}

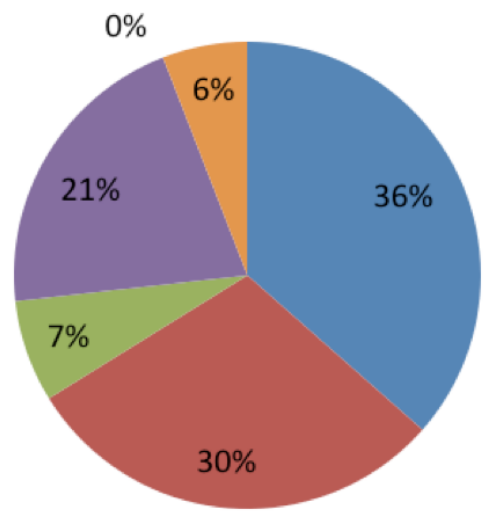

Medical staff (1 employed, 1 contracted)

- Radiology technician (2 employed, 1 contracted)

Nursing staff

Purchase of drugs

Tomography equipment

- Tomografy equipment maintenance

Chart 4. Percentage distribution of the costs for the routine management of the Nuclear Medicine unit.

This result is counterbalanced by an approximately $34 \%$ increase in the costs for a single patient service.

This model is believed to be valid also when considering the implementation of the new monograph chapter Compounding of Radiopharmaceuticals.

It appears to be self-evident how the implementation of a quality management system entails an increase in management costs. On the other hand, however, it also offers the possibility of initiating continuous quality improvement projects abating the risk indexes for adverse events that could involve patients, staff, and healthcare organizations themselves. Moreover, continuous process monitoring that has reached full maturity offers analytical data that can be used for devising quality improvement and optimization actions that can further improve the risk indexes and, at the same time, reduce healthcare expenditure.

\section{CONFLICT OF INTEREST}

The authors confirm that this article content has no conflict of interest.

\section{ACKNOWLEDGEMENTS}

Declared none.

\section{REFERENCES}

[1] Guide to Emergency Management - Planning in Health Care Joint Commission 2002

[2] Joint Commission International. Accreditation standards for ambulatory care. ed. 2010.

[3] Leape L.L. Reporting of adverse events. $N$ Engl J Med, 2002, 347(20) 1633-38.

[4] Reason J., Human errors: models and management. BMJ, 2000, 320, 768-70. 
[5] M. Marengo, Centri PET con ciclotrone: organizzazione del personale e costi di gestione. Corso Produzione di radionuclidi per gli impieghi medici. Scuola Superiore di Fisica in Medicina "P. Caldirola”, Napoli 17 novembre 2012.
[6] Ministero della Salute, Sicurezza dei pazienti e gestione del rischio clinico. Manuale per la formazione del personale sanitario. FNOMCeO, IPASVI, 2008.

[7] Vincent C., Understanding and responding to adverse events. $N$ Eng J Med, 2003, 348(11), 1051-6. 